\title{
Formulation of Scheme for Sports Training Management Based on System Dynamics
}

\author{
Bi Yao, JIA Ling, WANG Qingbin \\ Zhuhai College of Jilin University, Zhuhai, 519000 china
}

Key words: data mining; sports exercise; training decision; system dynamics

\begin{abstract}
In China, the sports exercise of college students is generally learned in sports class and mainly dominated by amateur workout, which is in a disordered state. This paper introduces the supporting system of aid decision making on sports training of college students established by adopting data warehousing technology and data mining technology to realize the organic integration in different aspects of college students and make scientific training theories and advanced training methods applied in sports exercise training management of college students, and the system is to produce new knowledge rules to enrich knowledge base with mining technology of data including sports performance and physical examination sheet, etc of college students; select corresponding model based on user input, and gradually generate a reasonable scheme for sports exercise training through combination of rules in knowledge base
\end{abstract}

\section{Introduction}

With rapid development of computer technology and thorough application in all aspects, especially development of artificial intelligent theory and data mining technology, the realization of organic integration in different aspects of college students and application of scientific training theory \# advanced training method to sports exercise training management of college students have become the possibility, and it is theoretically feasible to make data mining technology applied in the supporting system of aid decision making on sports training of college students. \$ This paper has found out a scientific and reasonable training scheme through research and analysis in all aspects including sports activity \# training of existing college students, etc and the combination of specific physical quality and health standard of college students, and tested the reasonability by means of application of aid scheme generated in sports class, so that the improvement for the existing supporting system on decision making may be made.

\section{Decision making based on system dynamics}

Basic elements of system dynamics

Basic elements constituting system dynamics model contain "flow" (Flow) and "element". "Flow" is divided into "material flow" (Material Flow" and "information flow" (Information Flow", while "element" includes "state variable" (Level), "rate" (Rate) and "auxiliary variable" (Auxiliary). Kinds of flow involves order flow, personnel flow, cash flow, equipment flow, logistics flow and information flow, all of which have concluded basic operation structures included in general organizations or enterprise operations. State variable denotes things or objects accumulated with passage of time in real world. In addition to tangible and visible state variables, such as inventory, number of people, money and gross of pollutant, etc, the intangible and invisible state variables are also contained, such as energy and pressure, etc. The value of state variable is decided by the rate controlling such state variable, and a state variable may be controlled by several rates. The rate could be divided into inflow rate and outflow rate, and the state variable is formed through accumulation of difference between inflow rate and outflow rate for a period. Auxiliary variable mainly has three meanings, in which the first denotes the process of data processing; the second denotes a certain environmental parameter value as a constant, and the third denotes input testing function or value of the system. The previous two conditions may be regarded as a part of rate, and the management control mechanism with a certain purpose is jointly formed by both rate and it; 
while the last condition is used for testing various different situations of model behavior. Modeling of system dynamics has three important components, including cause and effect feedback chart, flow chart and equation. Cause and effect feedback chart describes the cause-and-effect relationship between variables, which is an important tool in system dynamics; flow chart assists researchers to use symbols to represent the complex concept of model; structure of system dynamics model mainly consists of differential equations, and each equation between connecting state variable and rate means a differential equation. In the system dynamics, use finite differential equation for expression, and then solve each equation as time step to show the change of state in each time point of the system. There are three algorithms provided as estimations of change of state variable, respectively involving Euler's, 2nd-order Runge-Kutta and 4nd-order Runge-Kutta method. Each algorithm has advantages and disadvantages. It is necessary to select appropriate algorithm based on model structure.

Decision making of system dynamics

Research on data mining is integrated with technologies and achievements in several subjects fields, which makes the existing data mining method present diverse types. \& From the view of statistic analysis type, dada mining model used in statistic analysis technology has linear analysis and non-linear analysis \# regression analysis \# logistic regression analysis \# univariate analysis and multivariate analysis \# time series analysis \# the nearest series analysis \# the nearest neighbor algorithm, clustering analysis and other methods. \$ By taking advantages of these technologies, those data with abnormal patterns may be checked; then, these data could be explained with different statistic models and mathematical models used. What is explained is market rule and business opportunity hidden behind these data. Data mining technology for knowledge discovery is a kind of mining technology completely different from dada mining technology for statistic analysis, including artificial neuron network, decision-making tree of support vector machine, genetic algorithm, rough set rule, and discovery and association sequence, etc.

Analysis steps of system dynamics include:

(1) Problem identification.

(2) Confirm system boundary that means object and scope involved in system analysis.

(3) Establish cause and effect feedback chart, flow chart.

(4) Write out equation if system dynamics.

(5) Carry out simulation test and calculation, etc (Vensim software).

(6) Comparison, evaluation, policy analysis - discovery of the optimum system behavior Progress Chart of System Dynamics

Understanding of system dynamics on problem is obtained based on close reliable relationship between system behavior and internal mechanism and through establishment of mathematical model and manipulation of process to gradually explore the cause-and-effect relationship of change of form generated, and the system dynamics is also called as structure. So-called structure means a group of interlinked rule, tradition or policy, and such a group of structure decides characteristics of organization behavior. Data mining and problem solving system includes two modules, respectively including problem solving module and dada mining module. Data mining module is based on model provided by model base system. Carry out data mining for multi-dimensional data set in data warehouse to obtain necessary knowledge. Some special algorithms are required for data mining, such as association rule method, clustering algorithm and decision-making tree, etc. These algorithms have corresponding mining models in model base. As new knowledge and model, such result from data mining may be used for further enrichment of knowledge base and model base, and knowledge rules newly generated may provide decision support for physical education teaching reform. Problem solving module is the function module for processing of data in the forms input by user in man-machine interaction module, and the function of such function module is equivalent to that of inference machine in expert system. It is packaged in server with the form of data.

In the processing procedure, training programs suitable for participation of students may be obtained based on the matching of first components of knowledge rules between user input and knowledge base, so that personal training program and plan may be given. With implementation of user input, training programs of all users are grouped, and all results generated are summarized at 
the end of final input to generate a group training scheme to feed it back to users. The system for data warehouse management is designed for decision support not for transaction processing. Therefore, when user carries out data mining for each time, data useful for decision support are only extracted from operating environment and input in data warehouse. \$ After completion of input, data in data warehouse will be read-only and may be modified under special conditions.

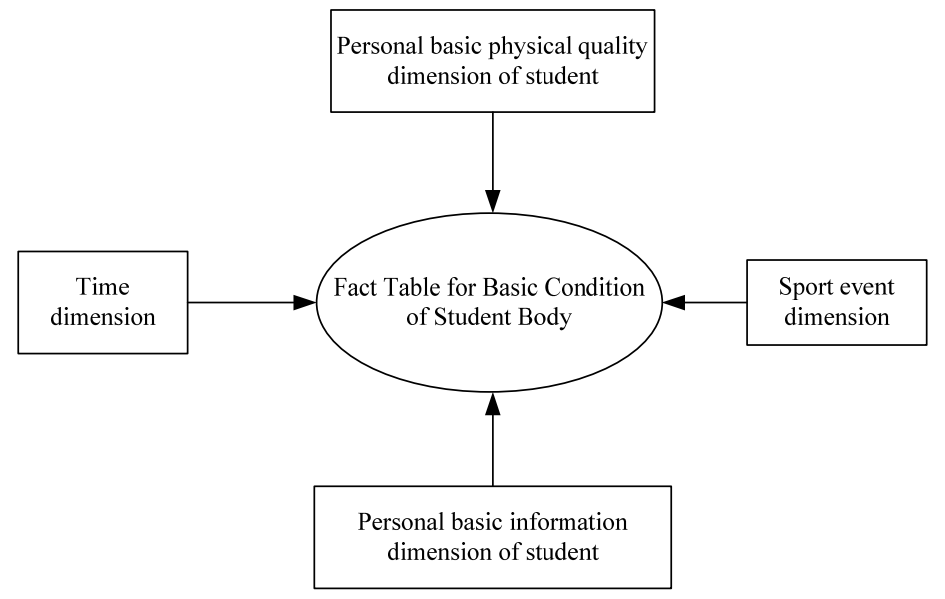

Fig.1 Star Type Model Figure

\section{Integration between system dynamics and database system}

If simulated parameters in system dynamics model are transferred into database system outside the model to become independent dada source, the simulated parameters and simulated results obtained from external database shall be stored in external database to realize the integration between system dynamics and database system in the simulation process. Zhang Guohua has used FoxPro to develop a suit of construction tool for system dynamics model with functions of database management, which is the modeling tool mainly dominated by DYNAMO language. It makes parameters in system and simulated results stored in the data file with xBase format. User may inquire about simulated results in virtue of narration of SQL language. Wu Dingfang [4] has researched data sources when taking linked external data sources (such as enterprise database and spreadsheet, etc) as simulation of system dynamics model; proposed to add component elements of "data sources" into model framework of traditional system dynamics; provided functions of model connectivity with external data sources, and developed the practical simulation tool software Maria according to model framework proposed. Guide dynamic thinking and cause-and-effect feedback loop concepts into expert system, and solve non-quantification problems not easily handled, uncertain problems unable to be mastered or fuzzy pattern problems in traditional system dynamics in virtue of characteristics of expert system. Xiao Naiyi has put forward the multi-dimensional simulation of expert system during implementation of integration research on system dynamics and regular inference mechanism to make system dynamics model may handle non-quantification variables. Complicated rules may be described in the variables of system dynamics model through regular inference of expert system. Bring in reliable elements of expert system to simulate decision making under undefined condition by system dynamics model. In addition, it is suggested to firstly use deduction framework written by other procedures and then use DYNAMO interface function for connection between system dynamics and expert system.

\section{Reference}

[1] Beynon M, Cosker D, Marshall D. An expert system for multi-criteria decision making using Dempster Shafer theory[J]. Expert Systems with Applications, 2001, 20(4):357-367.

[2] Van d K G, Van Heck E, De Vries H J, et al. Supporting Decision Making in Technology Standards Battles Based on a Fuzzy Analytic Hierarchy Process[J]. IEEE Transactions on 
Engineering Management, 2014, 61(2):336-348.

[3] Greco M, Barchiesi M A, Costa R. Enhancing Conflict Resolution through an AHP-Based Methodology[J]. International Journal of Management \& Decision Making, 2014, 13(1):17-41.

[4] Beynon M J. A method of aggregation in DS/AHP for group decision-making with the non-equivalent importance of individuals in the group[J]. Computers \& Operations Research, 2005, 32(7):1881-1896.

[5] Deniz Okul, Cevriye Gencer, Emel Kizilkaya Aydogan. A Method Based on SMAA-Topsis for Stochastic Multi-Criteria Decision Making and a Real-World Application[J]. International Journal of Information Technology \& Decision Making, 2014, 13(5):957-978. 